\title{
NEGOTIATING TRANSFER PRICING USING THE NASH BARGAINING SOLUTION
}

\author{
JULIO B. CLEMPNER ${ }^{a, *}$, ALEXANDER S. POZNYAK ${ }^{b}$
}

${ }^{a}$ School of Physics and Mathematics, National Polytechnic Institute

Luis Enrique Erro S/N, San Pedro Zacatenco, Del. Gustavo A. Madero, 07738 Mexico City, Mexico e-mail: julio@clempner.name

${ }^{b}$ Department of Control Automatics, Center for Research and Advanced Studies Av. IPN 2508, Col. San Pedro Zacatenco, 07360 Mexico City, Mexico e-mail: apoznyakectrl.cinvestav.mx

\begin{abstract}
This paper analyzes and proposes a solution to the transfer pricing problem from the point of view of the Nash bargaining game theory approach. We consider a firm consisting of several divisions with sequential transfers, in which central management provides a transfer price decision that enables maximization of operating profits. Price transferring between divisions is negotiable throughout the bargaining approach. Initially, we consider a disagreement point (status quo) between the divisions of the firm, which plays the role of a deterrent. We propose a framework and a method based on the Nash equilibrium approach for computing the disagreement point. Then, we introduce a bargaining solution, which is a single-valued function that selects an outcome from the feasible pay-offs for each bargaining problem that is a result of cooperation of the divisions of the firm involved in the transfer pricing problem. The agreement reached by the divisions in the game is the most preferred alternative within the set of feasible outcomes, which produces a profit-maximizing allocation of the transfer price between divisions. For computing the bargaining solution, we propose an optimization method. An example illustrating the usefulness of the method is presented.
\end{abstract}

Keywords: negotiated transfer pricing, Nash bargaining, tax avoidance, corporate taxation.

\section{Introduction}

1.1. Brief overview. Transfer pricing suggests the setting of the price at which a firm transfers goods and services between its collaborating (or not) divisions. It is employed as a profit allocation strategy to attribute a multinational corporation's net profit before tax when crossing international borders. Many firms implement the strategy of distributed products and services dispersed through numerous divisions at different locations. These divisions belong to more than one firm and the product passes through these different sites during its manufacturing/servicing stages (see Fig. 1) (Fredrickson, 1986; Ghosh et al., 2004; Karpowicz, 2012; Markides and Williamson, 1996; Wahab et al., 2016). This distributed strategy looks for different benefits: lower taxes, labor facilities regulations, lower costs, etc. However, for computing a maximum firm-wide profit

*Corresponding author surplus among divisions, several problems arise with international regulations. The arm-length rule enforces firms to establish pricing based on similar transactions done between divisions not of the same related firm but at an arm's length (OECD, 2010). Regulations on transfer pricing determine a 'fair' agreement concerning negotiation of the transfer price among divisions. The official definition of the arm's length standard reads: "a controlled transaction meets the arm's length standard if the results of the transaction are consistent with the results that would have been realized if uncontrolled taxpayers had engaged in the same transaction under the same circumstances."

In a multidivisional firm where divisions are required to transact with each other, the transfer price is used to determine costs. Transfer prices have a tendency to not be significantly different from the price established in the market for the reason that one of the divisions in such a transaction suffer the loss of money. The problem is that 


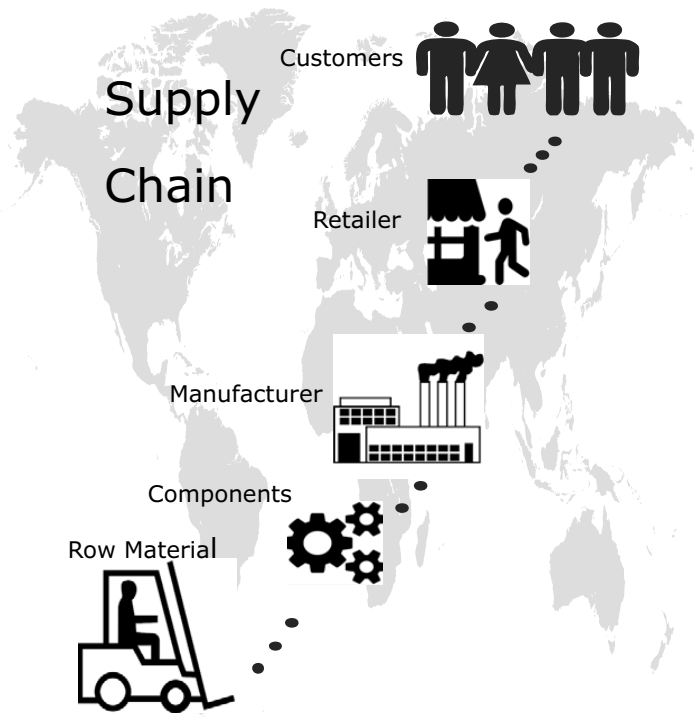

Fig. 1. Multidivisional firm.

if the firms start either buying for more than the prevailing performance market price or selling below the market price, this will affect their performance. In consequence, computing the optimal transfer price is a very interesting challenge that has attracted the attention of researchers from a wide range of disciplines, but it is still under debate among both practitioners and academics (Abdel-Khalik and Lusk, 1974; Alm, 2012; Beer and Loeprick, 2015; Devereux, 2007; Grabski, 1982; McAulay et al., 2001; Watson and Baumler, 1975).

Hirshleifer $(1956$; 1957) was the first in presenting analytical approaches to the transfer pricing problem finding a pricing structure for a mono-product firm involving a manufacturing and sales division, which identifies an efficient level of internal trade. Arrow (1959) as well as Baumol and Fabian (1964) extended Hirshleifer's model to settings of multiple products and divisions. Kanodia (1979) suggested how central management can construct transfer prices which allow risk sharing, making all the managers better off. Blois (1978) improved the model presented by Hirshleifer (1956; 1957), suggesting that even if the central management allows decentralization, then a large customer will be capable to enforce the transfer price rule of marginal cost upon its suppliers. There is earlier related literature on transfer pricing, considering a single period horizon (Enzer, 1975) or temporal stability (Burton et al., 1974). Enzer (1975) employed a linear programming method to solve the transfer pricing problem for obtaining an average price. Continuing Enzer's work, Jennergren (1977) proposed a solution for centralized decision making for the divisions. Thomas (1980) provided a review of the literature, considering psychological and empirical evidence for transfer pricing. He suggested that central management allows decentralization without scarifying the coordination associated with centralization. Thomas's idea coincided with the proposal of Dearden (1973) or Henderson and Dearden (1966). Amershi and Cheng (1990), Besanko and Sibley (1991) as well as Ronen and Balachandran (1988) solved the problem of transfer pricing considering that divisions are implicitly or explicitly subcontractors and with divisions paying a transfer price and incurring production costs. Rosenthal (2008) developed a cooperative game that provides transfer prices for the intermediate products (when valuation is known and when their valuations differ) in a vertically integrated supply chain, providing a solution that is fair and acceptable to all divisions. Leng and Parlarb (2012) extended the work of Rosenthal (2008), constructing a cooperative game based on computing the Shapley value-based transfer prices for a vertically integrated supply chain firm with an upstream division and multiple downstream divisions, which can independently determine their retail prices, and decide whether or not they will purchase from the upstream division at negotiated transfer prices.

Related work which solves transfer-price bargaining problems has been reported in the literature. Chalos and Haka (1990) suggested a bilateral bargaining methodology to study negotiated transfer-pricing outcomes between a buying and a selling division where each division had private profit information. Edlin and Reichelstein (1995) examined transfer price negotiations for solving a bilateral holdup problem in a multinational enterprise based on bargaining theory. They showed that information asymmetry will result in a transfer price outcome that is unfair and inefficient. Vaysman (1998) developed a bargaining model of negotiated transfer pricing incorporating private divisional information where the firm designs a compensation system employing divisional performance evaluation and negotiated transfer pricing. Haake and Martini (2013) analyzed two transfer-pricing schemes and their corresponding bargaining problems (Nash, 1950; Kalai and Smorodinsky, 1975) restricted to two divisions. In general, cooperative and noncooperative game theory solutions focusing on negotiations have been presented in the literature (Baldenius et al., 1999; Chwolka et al., 2010; Johnson, 2006; Wielenberg, 2000).

1.2. Main results. This paper analyzes and proposes a solution to the computational transfer pricing problem considering a firm consisting of several divisions. The main results are the following:

- We propose a solution for computing the transfer pricing problem from the point of view of the Nash bargaining game theory approach.

- In this negotiation process, divisions cooperate and 
all necessarily improve their position at the end of the process.

- Divisions operate over sequential transfers in which central management provides the transfer price decision that enables maximization of operating profits.

- The transfer pricing model involves costs and taxes.

- The division's unit production cost is dependent on the production quantity.

- The negotiation starts at the time that a division considers a disagreement point (status quo) which plays the role of a deterrent.

- We propose a framework and a method based on the Nash equilibrium approach for computing the disagreement point.

- The bargaining solution, which is a single-valued function, is the result of cooperation by the divisions.

- The final agreement is the most preferred alternative within the set of feasible outcomes which produces a profit-maximizing allocation of the transfer price between divisions.

- We propose an optimization for computing the bargaining solution method.

- The result of the optimization method is a simultaneous adjustment of quantity and the transfer price.

1.3. Organization of the paper. The paper is structured as follows. The next section presents the preliminaries, including Nash's bargaining game, and outlines the transfer pricing model. Section 3 describes the solution approach for the transfer pricing bargaining model. Section 4 suggests the transfer price bargaining solver. A numerical example showing the usefulness of the proposed approach is presented in Section 5. Finally, we close the paper in Section 6 with conclusions and an outline of future work.

\section{Preliminaries}

2.1. Bargaining. Nash's bargaining game (see Fig. 2) is based on a model in which players are assumed to negotiate on a set of feasible pay-offs (Trejo et al., 2017; Trejo and Clempner, 2018). A fundamental element of the game is the disagreement point (status quo) which plays the role of a deterrent. A bargaining solution is a single-valued function that selects an outcome from the feasible pay-offs for each bargaining problem, which is the result of cooperation by the players involved in the

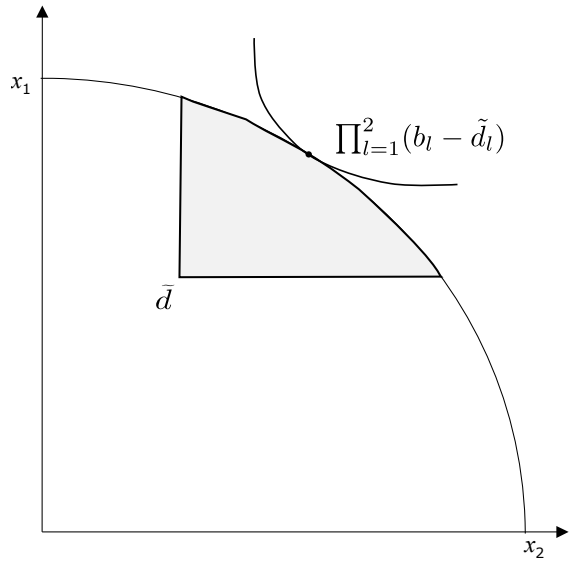

Fig. 2. Nash bargaining.

game. The agreement reached in the game is the most preferred alternative within the set of feasible outcomes.

The bargaining problem is described by the pair $(L, \tilde{d})$, where $L \subset \mathbb{R}^{n}$ is a set of feasible payoffs, $\tilde{d} \in$ $\mathbb{R}^{n}$ is a fixed disagreement vector and $l=1, \ldots, n$ is the number of players. We will call this the condensed form of the bargaining problem (see Forgó et al., 1999; Nash, 1950). It can be derived from the normal form of an $n$-person game $G=\left\{X^{1}, \ldots, X^{n} ; f_{1}, \ldots, f_{n}\right\}$ in a natural way. The set of all feasible payoffs (outcomes) is defined as $F=\left\{f: f=\left(f_{1}(x), \ldots, f_{n}(x)\right)\right\}, x \in X$ where $X=X^{1} \times \cdots \times X^{n}$. Given a disagreement vector $\tilde{d} \in \mathbb{R}^{n}$, the pair $(F, \tilde{d})$ is a bargaining problem in condensed form. We can derive another bargaining problem $(L, \tilde{d})$ from $G$ by extending the set of feasible outcomes $F$ to its convex hull $L$. Notice that any element $f \in L$ can be represented as $f=\sum_{l=1}^{n} \lambda_{l} f_{l}$, where $\left.\lambda f=\left(f_{1}(x(\lambda)), \ldots, f_{n}(x(\lambda))\right)\right), x \in X, \lambda_{l} \geq 0$ for all $l$, and $\sum_{l=1}^{n} \lambda_{l}=1$.

The payoff vector $f$ can be realized by playing the strategies $x^{l}$ with probability $\lambda^{l}$, and so $f$ is the expected payoff of the players. Thus, when the players face the bargaining problem, the question is which point of $L$ should be selected taking into account the different positions and strength of the players reflected in the set $L$ of extended payoffs and the disagreement point $\tilde{d}$.

Let $B$ denote the set of all pairs $(L, \tilde{d})$ such that (a) $L \subset \mathbb{R}^{n}$ is compact and convex, (b) there exists at least one $f \in L$ such that $f>\tilde{d}$. A Nash solution to the bargaining problem is a function $b: B \rightarrow \mathbb{R}^{n}$ such that $b(L, \tilde{d}) \in L$.

Proposition 1. There is a unique function $b$ such that for all $(L, \tilde{d}) \in B$ the vector $b(L, \tilde{d})=\left(b_{1}, \ldots, b_{n}\right)$ is a unique solution to the optimization problem 


$$
\begin{array}{ll}
\text { maximize } & g(b)=\prod_{l=1}^{n}\left(b_{l}-\tilde{d}_{l}\right) \\
\text { subject to } & b \in L, b \geq \tilde{d} .
\end{array}
$$

The objective function of problem in Eqn. (1) is usually called the Nash product.

2.2. Transfer pricing model. The proposed model is as follows (Rosenthal, 2008). We consider a multidivisional firm, which is a vertically integrated supply chain. We consider $n$ divisions and each division, is indexed by $l=1,2, \ldots, n$. Divisions jointly make their decisions to maximize the global profits of the firm. Along the supply chain, division $l$ sells $q^{l}$ units of intermediate goods on the market at price $\Delta_{p}^{l}$. The utility given by $\varphi=q^{l} \Delta_{p}^{l}$ is to be allocated among the $n$ divisions in the supply chain. In addition, the model takes into account the fact that intermediate goods involve acquisition and production costs $K$. Then, the profit of the $n$-th division is given by $\Delta_{p}^{l}-K^{l}$. The transfer pricing process involves divisions selling goods either externally at positive market price $p$ or internally to divisions at transfer price $\mathcal{P}$ such that the divisions' profit $\Delta_{p}$ is given by $\Delta_{p}=p-\mathcal{P}$.

We consider the case in which each level of the multidivisional firm consists of a single division. For $l=1, \ldots, n-1$, intermediate goods are shipped from level $l$ to level $l+1$, i.e., along the supply chain. Division $l$ sells intermediate goods to division $l+1$ at price $p^{l}$. Division $l+1$ sells intermediate goods to division $l+2$ at price $p^{l+1}$, and so on. Division $l$ makes its market pricing decision $p^{l}$ and sells $q^{l}\left(p^{l}\right)$ units of its intermediate goods to a given division $l$.

For $l=1, \ldots, n-1$, intermediate goods are shipped from level $l$ to level $l+1$, i.e., along the supply chain (see Fig. 1). Division $l$ makes its market pricing decision $p^{l}$ and sells $q^{l}\left(p^{l}\right)$ units of its intermediate goods to a given division $l$. Following Baldenius et al. (1999) as well as Leng and Parlarb (2012), division $l$ sales quantity $q^{l}$, which is determined by a linear demand function, i.e., $q^{l}\left(p^{l}\right)=\alpha^{l}-\beta^{l} p^{l}$, where $\alpha^{l}, \beta^{l}>0$ and $p^{l} \leq \alpha^{l} / \beta^{l}$. The model takes into account the fact that divisions are placed in different areas so that they have independent demands $q^{l}\left(p^{l}\right)$. Then, the division profit is given by

$$
\varphi^{1}\left(p^{1}, q^{1}\right)=p^{1} q^{1}\left(p^{1}\right)=\left(p^{1}-K^{1}\right)\left(\alpha^{1}-\beta^{1} p^{1}\right),
$$

where $K^{1}$ denotes acquisition and production costs, and

$$
\begin{aligned}
\varphi^{l}\left(p^{l}, q^{l}\right) & =p^{l} q^{l}\left(p^{l}\right) \\
& =\left(p^{l}-\mathcal{P}^{l}\right)\left(\alpha^{l}-\beta^{l} p^{l}\right), \quad l \geq 2,
\end{aligned}
$$

where $\mathcal{P}^{l}$ corresponds to the transfer price that division $l$ pays to division $l-1$ for $l \geq 2$. Thus Eqn. (3) can be written as

$$
\begin{aligned}
\varphi^{l}\left(p^{l}, p^{l-1}, q^{l}\right) & =\left(p^{l}-p^{l-1}\right) q^{l}\left(p^{l}\right) \\
& =\left(p^{l}-p^{l-1}\right)\left(\alpha^{l}-\beta^{l} p^{l}\right), \quad l \geq 2 .
\end{aligned}
$$

Owing of the existence of economies of scale, we assume that the division's unit production cost is dependent on the amount of production. Then, the unit production cost, which is incurred by division $l$ when the division sells $q^{l}\left(p^{l}\right)$ units of intermediate goods, is represented by $c^{l}\left(q^{l}\right)$. The amount of the division's total sales is $Q(\mathbf{q}) \equiv \sum_{l} q^{l}$, where $\mathbf{q}=\left(q^{1}\left(p^{1}\right), \ldots, q^{n}\left(p^{n}\right)\right)$. Then, the production cost can be written as $c(Q(\mathbf{q}))$. The corresponding effect on the divisions' costs is given by $\kappa_{c}^{l} c^{l}\left(q^{l}\right) q^{l}$, where $\kappa_{c}^{l} \in[0,1]$.

In addition, we represent the taxes that a division $l$ has to pay as a function depending on the good and the amount represented by $\tau^{l}\left(p^{l} q^{l}\right)$. We do not consider any specific function for the costs and the taxes, and we use the general form $c^{l}\left(q^{l}\right)$ and $\tau^{l}\left(p^{l} q^{l}\right)$ for our analysis. The corresponding effect on the divisions' costs is given by $\omega_{\tau}^{l} p^{l} q^{l}$, where $\omega_{\tau}^{l} \in[0,1]$.

The model involves the following variables:

- market prices $p^{l}$ for one intermediate good (sold from $l$ to $l+1)$

- amounts $q^{l}$ of intermediate good $l$ shipped from $l$ to $l+1$ for $l=1, \ldots, n-1$;

- production costs,

$$
c^{l}(Q(\mathbf{q})):=\kappa_{c}^{l} c^{l}\left(q^{l}\right) q^{l}, \quad \kappa_{c}^{l} \in[0,1]
$$

(e.g., transactional costs, raw materials, components, and their per period inventory costs in dollars) at each level $l=\overline{1, n}$;

- taxes,

$$
\tau^{l}\left(p^{l} q^{l}\right):=\omega_{\tau}^{l} p^{l} q^{l}, \quad \omega_{\tau}^{l} \in[0,1]
$$

at each level $l=\overline{1, n}$.

Remark 1. The taxes $\tau^{l}\left(p^{l} q^{l}\right)$ can be eliminated from the computation process taking $\omega_{\tau}^{l}=0$ if it is the case that a multidivisional firm does not use transfer pricing as a tool for reducing the firm's total tax payment.

We suppose that all divisions are located in different marketing areas. Therefore, they face independent demands $q^{l}$, costs $c^{l}\left(q^{l}\right)$ and taxes $\tau^{l}\left(p^{l} q^{l}\right)$. Then, the division's utility $\Pi^{l}$ for each level $l=\overline{1, n}$ is given by

$$
\begin{aligned}
\varphi^{1}\left(p^{1}, q^{1}\right)= & \left(p^{1}-K^{1}\right) q^{1}-c^{l}(Q(\mathbf{q}))-\tau^{1}\left(p^{1} q^{1}\right), \\
\varphi^{l}\left(p^{l}, p^{l-1}, q^{l}\right)= & \left(p^{l}-p^{l-1}\right) q^{l}-c^{l}(Q(\mathbf{q})) \\
& -\tau^{l}\left(p^{l} q^{l}\right),
\end{aligned}
$$




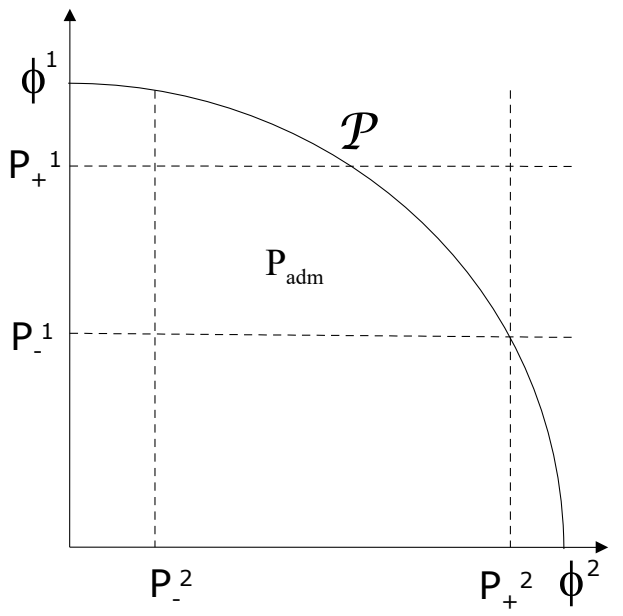

Fig. 3. Bounds of price.

where $l=1$ represents the first division and $l=2, \ldots, n$ the rest of the divisions on the vertically integrated supply chain.

Our model considers a centralized structure allowing divisions to act necessarily cooperatively. The Pareto set can be defined as (Clempner and Poznyak, 2015; 2017; Clempner, 2016)

$$
\begin{aligned}
\mathcal{P}:= & \left\{x^{*}(\lambda):=\arg \max _{x \in X_{\mathrm{adm}}=P_{\mathrm{adm}} \otimes Q_{\mathrm{adm}}} J(x \mid \lambda),\right. \\
& \left.\lambda \in \mathcal{S}^{n}\right\},
\end{aligned}
$$

where

$$
\begin{gathered}
J(x \mid \lambda):=\lambda_{1} \varphi^{1}\left(p^{1}, q^{1}\right)+\sum_{l=2}^{n} \lambda_{l} \varphi^{l}\left(p^{l}, p^{l-1}, q^{l}\right), \\
x=\left\{(p, q): p=\left(p^{1}, \ldots, p^{n}\right)^{\top}\right. \text { and } \\
\left.q=\left(q^{1}\left(p^{1}\right), \ldots, q^{n}\left(p^{n}\right)\right)^{\top}\right\} \\
\mathcal{S}^{n}:=\left\{\lambda \in \mathbb{R}^{n}: \lambda_{l} \in[0,1], \sum_{l=1}^{n} \lambda_{l}=1\right\},
\end{gathered}
$$

with the Pareto front given by

$$
\begin{gathered}
\Psi\left(x^{*}(\lambda)\right)=\left(\varphi^{1}\left(x^{*}(\lambda)\right), \varphi^{2}\left(x^{*}(\lambda)\right), \ldots,\right. \\
\left.\varphi^{n}\left(x^{*}(\lambda)\right)\right) .
\end{gathered}
$$

Bounds to $p^{l}$ and $q^{l}$ determine the maximum and minimum transfer prices legally authorized. Bounds are established by the arm's length price as well as the quantity of goods traded. The bounds (Figs. 3 and 4 ) determine a specific decision area where the optimal strategies can be selected,

$$
\begin{gathered}
p^{l} \in\left[p_{-}^{l}, p_{+}^{l}\right], \quad q^{l} \in\left[q_{-}^{l}, q_{+}^{l}\right], \\
P_{\mathrm{adm}}:=\bigcap_{l=1}^{n}\left[p_{-}^{l}, p_{+}^{l}\right], \quad Q_{\mathrm{adm}}:=\bigcap_{l=1}^{n}\left[q_{-}^{l}, q_{+}^{l}\right] .
\end{gathered}
$$

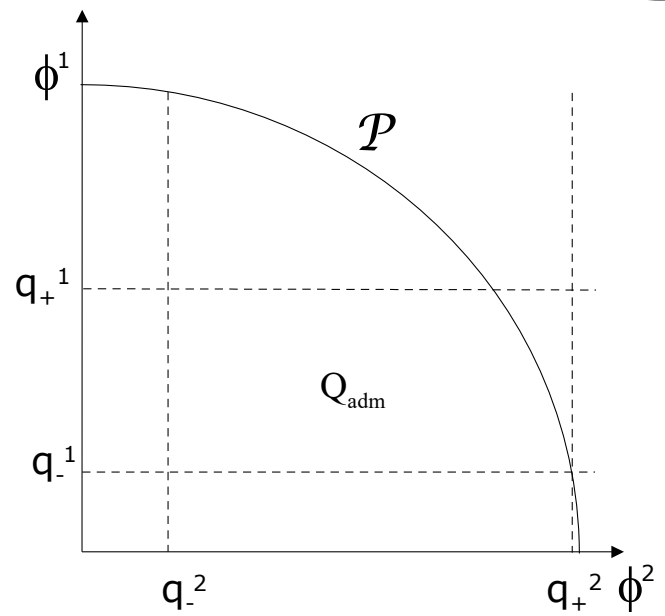

Fig. 4. Bounds of quantity.

\section{Transfer pricing Nash bargaining solution}

We start with some notation (Clempner and Poznyak, 2011; 2016; Trejo et al., 2015). For a finite set of divisions (players) $\mathcal{N}$ with $n$ elements, let $\mathbb{R}^{n}$ denote the $n$-dimensional Euclidean space with coordinates indexed by $l=1, \ldots, n$. Any point in $X \subseteq \mathbb{R}^{n}$, called the joint strategy of the divisions, is denoted by $x=\left(x^{l}\right)_{l \in \mathcal{N}}$, and also by $x=\left(x^{1}, \ldots, x^{n}\right), l=\overline{1, n}$. The set $X$ is a convex and compact set. For $l \in \mathcal{N}$ and $x=\left(x^{l}\right)_{l \in \mathcal{N}} \in X, \hat{x}$ denotes the $(n-1)$-dimensional vector constructed from $x$ by deleting the $l$-th coordinate $x^{l}$. Here $x^{\hat{l}}$ is a strategy of the rest of the players adjoint to $x^{l}$, namely,

$$
\begin{aligned}
x^{\hat{l}} & :=\left(x^{1}, \ldots, x^{l-1}, x^{l+1}, \ldots, x^{n}\right)^{\top} \\
& \in X^{\hat{l}}:=\bigotimes_{m=1, m \neq l}^{n} X^{m} .
\end{aligned}
$$

The point $x$ is written as $\left(x^{l}, x^{\hat{l}}\right)$.

An $n$-division game is defined by a triplet $\Gamma=$ $\left(\mathcal{N},\left\{A_{l}\right\}_{l \in \mathcal{N}},\left\{\varphi_{l}\right\}_{l \in \mathcal{N}}\right)$, where $\mathcal{N}$ is the set of divisions and each $A_{l}(l \in \mathcal{N})$ is finite set of division l's actions. The Cartesian product $A=\bigotimes_{l=1}^{n} A^{l}$ is the set of action profiles $a=\left(a^{1}, \ldots, a^{n}\right)$ for $n$ players. The utility function $\varphi_{l}$ of division $l$ is a real-valued function on $A$. Each player $l \in \mathcal{N}$, for a given a strategy $x^{l}$, gets the utility

$$
\varphi_{l}(x)=\sum_{x^{1} \in X^{1}} \cdots \sum_{x^{n} \in X^{n}} \varphi_{l}\left(a^{1}, \ldots, a^{n}\right) \prod_{l=1}^{n} x^{l}\left(a^{l}\right)
$$

where $x^{l}\left(a^{l}\right)$ is the strategy of the action $a^{l}$.

Divisions try to reach one of the Nash equilibria, that is, each division $l$ tries to find a joint strategy $x^{*}=$ 
$\left(x^{1 *}, \ldots, x^{n *}\right) \in X$ satisfying, for any admissible $x^{l} \in$ $X^{l}$ and any $l=\overline{1, n}$,

$$
\begin{aligned}
& \varphi_{l}\left(x^{l}, x^{\hat{l}}\right)-\max _{x^{l} \in X^{l}} \varphi_{l}\left(x^{l}, x^{\hat{l}}\right) \leq 0 \\
& \qquad \text { for any } x^{l} \in X^{l} \text { and all } l=\overline{1, n},
\end{aligned}
$$

where $\hat{x}(x)=\left(x^{\hat{1} \top}, \ldots, x^{\hat{n} \top}\right)^{\top} \in \hat{X} \subseteq \mathbb{R}^{n(n-1)}$ and $p \geq 1$ (Tanaka and Yokoyama, 1991; Tanaka, 1989). Here $\varphi_{l}\left(x^{l}, x^{\hat{l}}\right)$ is the utility function of player $l$, who plays strategy $x^{l} \in X^{l}$ while the rest of the players strategy $x^{\hat{l}} \in X^{\hat{l}}$

Consider

$$
\begin{aligned}
& G_{L_{p}}(x, \hat{x}(x)) \\
& :=\left[\sum_{l=1}^{n}\left|\left(\max _{x^{l} \in X^{l}} \varphi_{l}\left(x^{l}, x^{\hat{l}}\right)\right)-\varphi_{l}\left(x^{l}, x^{\hat{l}}\right)\right|^{p}\right]^{1 / p} .
\end{aligned}
$$

If we define the utopia point

$$
\bar{x}^{l}:=\arg \max _{x^{l} \in X^{l}} \varphi_{l}\left(x^{l}, x^{\hat{l}}\right),
$$

then, substituting (10) in (9), the original problem given can be rewritten as

$$
\begin{aligned}
G_{L_{p}}(x, \hat{x}(x)) & \\
& :=\left[\sum_{l=1}^{n}\left|\varphi_{l}\left(\bar{x}^{l}, x^{\hat{l}}\right)-\varphi_{l}\left(x^{l}, x^{\hat{l}}\right)\right|^{p}\right]^{1 / p} .
\end{aligned}
$$

The functions $\varphi_{l}\left(x^{l}, x^{\hat{l}}\right)(l=\overline{1, n})$ are assumed to be concave in all their arguments.

Remark 2. The function $G_{L_{p}}(x, \hat{x}(x))$ satisfies the Nash property

$$
\varphi_{l}\left(x^{l}, x^{\hat{l}}\right)-\varphi_{l}\left(\bar{x}^{l}, x^{\hat{l}}\right) \leq 0
$$

for any $x^{l} \in X^{l}$ and all $l=\overline{1, n}$.

If the function $G_{L_{p}}(x, \hat{x}(x))$ is strictly concave and the Hessian matrix is negative semi-definite, then $G_{L_{p}}(x, \hat{x}(x))$ attains a maximum at $(x, \hat{x}(x))$ and satisfies (Trejo et al., 2015)

$$
\begin{aligned}
\nabla^{2} G_{L_{p}}(x, \hat{x}(x)) & {\left[\begin{array}{lll}
\frac{\partial^{2}}{\left(\partial x_{1}\right)^{2}} G_{L_{p}}(x, \hat{x}(x)) & \ldots \\
\frac{\partial^{2}}{\partial x_{2} \partial x_{1}} G_{L_{p}}(x, \hat{x}(x)) & \ldots \\
\ldots \ldots \ldots \ldots & \ldots \ldots \ldots \\
\frac{\partial^{2}}{\partial x_{n} \partial x_{1}} G_{L_{p}}(x, \hat{x}(x)) & \ldots
\end{array}\right.}
\end{aligned}
$$

$$
\begin{aligned}
& \frac{\partial^{2}}{\partial x_{1} \partial x_{n}} G_{L_{p}}(x, \hat{x}(x)) \\
& \frac{\partial^{2}}{\partial x_{2} \partial x_{n}} G_{L_{p}, \delta}(x, \hat{x}(x)) \\
& \frac{\partial^{2}}{\left(\partial x_{n}\right)^{2}} G_{L_{p}}(x, \hat{x}(x)) \\
& =\left[\begin{array}{cc}
\delta I_{n_{1} \times n_{1}} & D G_{1,2}\left(\hat{u}_{1,2}\right) \\
D G_{2,1}\left(\hat{u}_{2,1}\right) & \delta I_{n_{2} \times n_{2}} \\
\ldots \ldots \ldots \ldots \ldots \ldots \ldots \ldots & \ldots \ldots \ldots \\
D G_{3,1}\left(\hat{u}_{3,1}\right) & D G_{3,2}\left(\hat{u}_{3,2}\right)
\end{array}\right. \\
& \ldots D G_{1, \mathcal{N}}\left(\hat{u}_{1, \mathcal{N}}\right) \\
& \text {.. } D G_{3,2}\left(\hat{u}_{3,2}\right) \\
& \begin{array}{ll}
\cdots & \ldots \ldots \\
\cdots & \delta I_{n_{n} \times n_{n}}
\end{array} \\
& <0
\end{aligned}
$$

or, equivalently, $\delta$ should provide the inequality

$$
\delta>\max _{x \in X}\left[\Lambda_{\max }\left(\nabla^{2} G_{L_{p}}(x, \hat{x}(x))\right)\right],
$$

where $\Lambda_{\max }$ is the maximum eigenvalue.

The bargaining game $\Gamma$ is based on a model in which players are assumed to negotiate on a set of feasible pay-offs $\Phi$. A fundamental element of the game is the disagreement point $f_{l}^{*}$ (status quo), which plays the role of a deterrent. A bargaining solution is a single-valued function that selects an outcome from the feasible pay-offs for each bargaining problem. The agreement reached in the game is the most preferred alternative within the set of feasible outcomes $\Phi$. Nash (1950) proposed this approach by presenting four axioms and showing that they characterize the Nash bargaining solution.

Definition 1. Nash bargaining transfer pricing. For a finite set of divisions $\mathcal{N}$ with $n$ elements of a game $\Gamma$, a strategy $x^{*}$ is called a Nash bargaining solution for the transfer price of $\Gamma$ if $x^{*}$ is an optimal solution of the maximization problem

$$
\prod_{l=1}^{n}\left(\varphi_{l}\left(x^{l}, x^{\hat{l}}\right)-\tilde{f}_{l}\left(\tilde{x}^{l}, \tilde{x}^{\hat{l}}\right)\right) \rightarrow \max _{x \in X}
$$

subject to

$$
\begin{gathered}
x \in \Phi, \\
\varphi_{l}(x) \geq \tilde{f}_{l}(\tilde{x}) \quad \text { for all } l=1, \ldots, n,
\end{gathered}
$$

where $\varphi$ is the payoff and $\tilde{f}_{l}$ is the disagreement point. The pay-off $\Phi\left(x^{*}\right)=\left(\varphi_{l}\left(x^{*}\right)\right)_{l=\overline{1, n}}$ of divisions generated by the Nash bargaining solution $x^{*}$ is the bargaining solution payoff. 
Let $\alpha^{n}=\left(\alpha_{l}\right)_{l=\overline{1, n}}$. Then, we rewrite the problem (14) as follows:

$$
g(x)=\sum_{l=1}^{n} \alpha_{l} \log \left(\varphi_{l}\left(x^{l}, x^{\hat{l}}\right)-\tilde{f}_{l}\left(\tilde{x}^{l}, \tilde{x}^{\hat{l}}\right)\right) \rightarrow \max _{x \in X}
$$

subject to

$$
\varphi_{l}(x, \hat{x}(x))>f_{l}^{*}
$$

where $\alpha_{l}$ is called the individual weight of each division such that $\sum_{l=1}^{n} \alpha_{l}=1, \tilde{f}_{l}$ is the disagreement point ( $l=$ $\overline{1, n})$ or the status-quo and $\tilde{x}=\left(\tilde{x}^{l *}, \tilde{x}^{\hat{l}}\right)$ is the status-quo strategy.

Specifically, we will consider the disagreement point as a division trying to reach one of the $L_{p}$-Nash equilibria,

$$
\tilde{f}_{l}\left(\tilde{x}^{l *}, \tilde{x}^{\hat{l}}\right)=\left[\sum_{l=1}^{n}\left|\varphi_{l}\left(\overline{\tilde{x}}^{l *}, \tilde{x}^{\hat{l}}\right)-\varphi_{l}\left(x^{l}, x^{\hat{l}}\right)\right|^{p}\right]^{1 / p} .
$$

\section{Transfer price bargaining solver with additional constraints}

Let us introduce the "slack" vectors $c \in \mathbb{R}^{n}$ with nonnegative components, that is, $c_{j} \geq 0$ for all $j=$ $1, \ldots, n$. The original problem (9) can be rewritten as

$$
\begin{array}{r}
\{g(x, \hat{x}(x))\} \rightarrow \max _{x \in X_{\mathrm{adm}}, c \geq 0} \\
X_{\mathrm{adm}}:=\left\{x \in X: x \geq 0, A_{\mathrm{eq}} x=b_{\mathrm{eq}},\right. \\
\left.A_{\text {ineq }} x-b_{\text {ineq }}+c=0\right\}
\end{array}
$$

Notice that this problem may have a non-unique solution and $\operatorname{det}\left(A_{\mathrm{eq}}^{\mathrm{\top}} A_{\mathrm{eq}}\right)=0$. Define by $X^{*} \subseteq X_{\text {adm }}$ the set of all solutions of the problem $15 \mathrm{~b}$.

Consider the penalty function given by

$$
\begin{aligned}
\tilde{\mathcal{V}}_{k}(x, c):= & \mu\{g(x, \hat{x}(x))\} \\
& -k\left[\frac{1}{2}\left\|A_{\mathrm{eq}} x-b_{\mathrm{eq}}\right\|^{2}\right. \\
& \left.+\frac{1}{2}\left\|A_{\text {ineq }} x-b_{\text {ineq }}+c\right\|^{2}\right],
\end{aligned}
$$

where the parameters $k$ and $c$ are positive. Notice also that

$$
\begin{aligned}
& \arg \max _{x \in X_{\text {adm }}, c \geq 0} \tilde{\mathcal{V}}_{k, \delta}(x, c) \\
&=\arg \max _{x \in X_{\text {adm }}, c \geq 0} \mathcal{V}_{\mu, \delta}(x, c),
\end{aligned}
$$

where $\mu:=k^{-1}>0$ and

$$
\begin{aligned}
\mathcal{V}_{\mu, \delta}(x, c) \\
:=\mu\{g(x, \hat{x}(x))\}-\frac{1}{2}\left\|A_{\text {eq }} x-b_{\mathrm{eq}}\right\|^{2} \\
\quad-\frac{1}{2}\left\|A_{\text {ineq }} x-b_{\text {ineq }}+c\right\|^{2} \\
\quad-\frac{\delta}{2}\left(\|x\|^{2}+\|\hat{x}\|^{2}+\|c\|^{2}\right) .
\end{aligned}
$$

Obviously, the optimization problem

$$
\tilde{\mathcal{V}}_{\mu, \delta}(x, c) \rightarrow \max _{x \in X_{\text {adm }}, c \geq 0}
$$

has a unique solution since the optimized function (16) is strongly convex (Poznyak, 2008) if $\delta>0$. The idea of the penalty functions method (PFM) consists in the following idea: If the penalty parameter $\mu$ and the regularizing parameter $\delta$ tend to zero in a particular manner, then we may expect that $x^{*}(\mu, \delta)$ and $c^{*}(\mu, \delta)$, which are the solutions to the optimization problem

$$
\mathcal{V}_{\mu, \delta}(x, c) \rightarrow \max _{x \in X_{\text {adm }}, c \geq 0},
$$

tend to the set $V^{*}$ of all solutions of the original optimization problem (15b), that is,

$$
\rho\left\{x^{*}(\mu, \delta), c^{*}(\mu, \delta) ; X^{*}\right\} \underset{\mu, \delta \downarrow 0}{\longrightarrow} 0,
$$

where $\rho\left\{a ; X^{*}\right\}$ is the Hausdorff distance defined as

$$
\rho\left\{a ; X^{*}\right\}=\min _{x \in X^{*}}\left\|a-x^{*}\right\|^{2} .
$$

Below we define exactly how the parameters $\mu$ and $\delta$ should tend to zero to provide the property (19).

Then, if we assume that

(a) the bounded set $X^{*}$ of all solutions of the original optimization problem (15b) is not empty and Slater's condition holds, that is, there exists a point $\stackrel{\circ}{x} \in X_{\text {adm }}$ such that

$$
A_{\text {ineq }} \stackrel{\circ}{x}<b_{1},
$$

(b) the parameters $\mu$ and $\delta$ are time-varying, i.e.,

$$
\mu=\mu_{n}, \quad \delta=\delta_{n}(n=0,1,2, \ldots,)
$$

such that

$$
0<\mu_{n} \downarrow 0, \quad \frac{\mu_{n}}{\delta_{n}} \downarrow 0 \quad \text { as } n \rightarrow \infty,
$$

then

$$
\begin{aligned}
& x_{n}^{*}:=x^{*}\left(\mu_{n}, \delta_{n}\right) \underset{n \rightarrow \infty}{\longrightarrow} x^{* *}, \\
& c_{n}^{*}:=c^{*}\left(\mu_{n}, \delta_{n}\right) \underset{n \rightarrow \infty}{\longrightarrow} c^{* *},
\end{aligned}
$$

where $x^{* *} \in X^{*}$ is the solution to the original problem (15b) with the minimal weighted norm, i.e.,

$$
\left\|x^{* *}\right\| \leq\left\|x^{*}\right\| \quad \text { for all } x^{*} \in X^{*}
$$

and

$$
c^{* *}=b_{1}-A_{1} x^{* *} \text {. }
$$


The format version $(n=0,1, \ldots)$ of the proximal method with some fixed admissible initial values $\left(x_{0} \in\right.$ $\left.X, \hat{x}_{0}(x) \in \hat{X}, c_{0} \geq 0\right)$ is as follows:

$$
\begin{aligned}
x_{n+1}= & \arg \max _{x \in X}\left\{-\frac{1}{2}\left\|x-x_{n}\right\|^{2}\right. \\
& \left.+\gamma \mathcal{V}_{\mu_{n}, \delta_{n}}\left(x, \hat{x}_{n}(x), c_{n}\right)\right\}, \\
\hat{x}_{n+1}(x)= & \arg \max _{\hat{x} \in \hat{X}}\left\{-\frac{1}{2}\left\|\hat{x}(x)-\hat{x}_{n}(x)\right\|^{2}\right. \\
& \left.+\gamma \mathcal{V}_{\mu_{n}, \delta_{n}}\left(x_{n}, \hat{x}(x), c_{n}\right)\right\},
\end{aligned}
$$

where $\mathcal{V}_{\mu_{n}, \delta_{n}}(x, \hat{x}(x), c)$ is given by

$$
\begin{aligned}
\mathcal{V}_{\mu_{n}, \delta_{n}}( & \left.x, \hat{x}_{n}(x), c\right) \\
:= & \mu g\left(x, \hat{x}_{n}(x)\right) \\
& -\frac{1}{2}\left(A_{\text {ineq }} x-b_{\text {ineq }}+c\right) \\
& -\frac{\delta}{2}\left(\|x\|^{2}+\|\hat{x}\|^{2}+\|c\|^{2}\right) \\
= & \mu g\left(x, \hat{x}_{n}(x)\right)-\frac{1}{2}\left(A_{\text {ineq }} x-b_{\text {ineq }}+c\right) \\
& -\frac{\delta}{2}\left(\|x\|^{2}+\|\hat{x}\|^{2}+\|c\|^{2}\right) .
\end{aligned}
$$

Then, developing, we have

$$
\begin{aligned}
x_{n+1}= & -\frac{1}{2}\left\|x-x_{n}\right\|^{2}+\gamma \mathcal{V}_{\mu_{n}, \delta_{n}}\left(x, \hat{x}_{n}(x), c_{n}\right) \\
= & -\frac{1}{2}\left\|x-x_{n}\right\|^{2}+\gamma\left[\mu g\left(x, \hat{x}_{n}(x)\right)\right. \\
& -\frac{1}{2}\left(A_{\text {ineq }} x-b_{\text {ineq }}+c\right) \\
- & \left.\frac{\delta}{2}\left(\|x\|^{2}+\|\hat{x}\|^{2}+\|c\|^{2}\right)\right] . \\
\bar{x}_{n+1}(x)= & -\frac{1}{2}\left\|\hat{x}(x)-\hat{x}_{n}(x)\right\|^{2} \\
& +\gamma \mathcal{V}_{\mu_{n}, \delta_{n}}\left(x_{n}, \hat{x}(x), c_{n}\right) \\
= & -\frac{1}{2}\left\|\hat{x}(x)-\hat{x}_{n}(x)\right\|^{2}+\gamma\left[\mu g \left(x_{n}, \hat{x}(x)\right.\right. \\
& -\frac{1}{2}\left(A_{\text {ineq }} x-b_{\text {ineq }}+c\right) \\
& \left.-\frac{\delta}{2}\left(\|x\|^{2}+\|\hat{x}\|^{2}+\|c\|^{2}\right)\right] .
\end{aligned}
$$

\section{Numerical example}

5.1. Problem description. Jemae is a fictional large multi-divisional corporation which manufactures parts of vehicles in three different countries and ensembles the cars in the USA, spending money on distribution and marketing costs. Each divisions is responsible for its own profits. The firm considers this situation a disagreement point because divisions often transact with each other as if transacting with an outside customer, impacting significantly the global profits of the company. In order to solve the problem, the company is looking to coordinate the transfer pricing of the divisions. The profit maximization of the multinational group will look at where the tax rates are lower and seek to put more profit there. In this example, we are considering different production costs and tax rates for each division. The goal is to establish the transfer prices for each division based on the bargaining Nash solution, providing incentives for divisional managers to act in the best interest of the corporation as a whole.

For solving the problem, we employ the proposed computational solver approach to find the bargaining equilibrium point. Finally, we perform an analysis to study the economic variables for the price and quantity.

The rest of this section is divided into two parts. In the first one, we describe the solution method. In the second, we present the parameters used for our computations and discuss the numerical results.

5.2. Solution method. For solving the problem, we employ the proximal method given by

$$
x_{n+1}=\arg \max _{x \in X_{\text {adm }}}\left\{-\frac{1}{2}\left\|x-x_{n}\right\|^{2}+\gamma g(x)\right\},
$$

where $\gamma$ is a positive scalar. We will assume that each $f(x): \mathbb{R}^{n} \rightarrow \mathbb{R}$ is a convex function and $X_{\text {adm }}$ is a nonempty closed convex set. We introduce a regularization method as follows:

$$
\begin{aligned}
\mathcal{V}_{\mu, \delta} & (x, c) \\
:= & \mu\{g(x, \hat{x}(x))\}-\frac{1}{2}\left\|A_{\text {ineq }} x-b_{\text {ineq }}+c\right\|^{2} \\
& -\frac{\delta}{2}\left(\|x\|^{2}+\|\hat{x}\|^{2}+\|c\|^{2}\right) .
\end{aligned}
$$

Tikhonov's regularization is one of the most popular approaches to solve discrete ill-posed minimization problems. The method aims at establishing an approximation of $x$ by replacing the minimization of an ill-posed problem.

For the transfer pricing bargaining problem, we have

$$
\begin{aligned}
g(x)= & \sum_{l=1}^{n} \alpha_{l} \log \left(\varphi_{l}\left(x^{l}, x^{\hat{l}}\right)\right. \\
& \left.-f_{l}^{*}\left(x^{l *}, x^{\hat{l} *}\right)\right) \rightarrow \max _{x \in X}
\end{aligned}
$$

subject to

$$
\varphi_{l}(x, \hat{x}(x))>f_{l}^{*},
$$

where

$$
\begin{aligned}
\varphi_{1} & =\left[\left(p^{1}-K^{1}\right) q^{1}-\kappa_{c}^{1} c^{1} q^{1}-\omega_{\tau}^{1} p^{1} q^{1}\right], \\
\varphi_{2} & =\left[\left(p^{2}-p^{1}\right) q^{2}-\kappa_{c}^{2} c^{2} q^{2}-\omega_{\tau}^{2} p^{2} q^{2}\right], \\
\varphi_{3} & =\left[\left(p^{3}-p^{2}\right) q^{3}-\kappa_{c}^{3} c^{3} q^{3}-\omega_{\tau}^{3} p^{3} q^{3}\right], \\
\varphi_{4} & =\left[\left(p^{4}-p^{3}\right) q^{4}-\kappa_{c}^{4} c^{4} q^{4}-\omega_{\tau}^{4} p^{4} q^{4}\right] .
\end{aligned}
$$


and

$$
A_{\text {ineq }}=\left[\begin{array}{cccccccc}
1 & 0 & 0 & 0 & 0 & 0 & 0 & 0 \\
0 & 1 & 0 & \cdot & \cdot & 0 & 0 & 0 \\
\cdot & \cdot & 1 & \cdot & \cdot & \cdot & \cdot & \cdot \\
\cdot & & \cdot & 1 & \cdot & \cdot & \cdot & \cdot \\
\cdot & & & \cdot & 1 & 0 & \cdot & \cdot \\
0 & \cdot & \cdot & \cdot & 0 & 1 & 0 & \cdot \\
0 & \cdot & \cdot & \cdot & \cdot & \cdot & 1 & 0 \\
0 & \cdot & \cdot & \cdot & \cdot & \cdot & 0 & 1 \\
-1 & 0 & \cdot & \cdot & \cdot & 0 & 0 & 0 \\
0 & -1 & \cdot & \cdot & \cdot & \cdot & 0 & 0 \\
\cdot & \cdot & -1 & \cdot & \cdot & \cdot & \cdot & \cdot \\
\cdot & \cdot & \cdot & -1 & \cdot & \cdot & \cdot & \cdot \\
\cdot & \cdot & \cdot & \cdot & -1 & \cdot & \cdot & \cdot \\
0 & \cdot & \cdot & \cdot & 0 & -1 & 0 & 0 \\
0 & \cdot & \cdot & \cdot & \cdot & -1 & 0 \\
0 & 0 & \cdot & \cdot & \cdot & \cdot & -1
\end{array}\right],
$$

5.3. Solution of the transfer price problem. The bounds are defined as follows: $p^{l} \in P_{\mathrm{adm}}:=\left[p_{-}^{l}, p_{+}^{l}\right]$, $q^{l} \in Q_{\mathrm{adm}}:=\left[q_{-}^{l}, q_{+}^{l}\right]$. For each player $l=1, \ldots, 4$, we have $p^{1} \in[85000,140000], p^{2} \in[70000,150000], p^{3} \in$ [90000, 160000], $p^{4} \in[80000,190000], q^{1} \in[650,800]$, $q^{2} \in[800,1100], q^{3} \in[650,1200], q^{4} \in[700,1600]$.

Production costs (e.g., transactional costs, raw materials, components, and their per period inventory costs in dollars) are determined by $c^{l}\left(q^{l}\right):=\kappa_{c}^{l} q^{l}, \kappa_{c}^{l} \geq 0$. For each player $l=1, \ldots, 4$, we have $\kappa_{c}^{1}=0.1, \kappa_{c}^{2}=$ $0.02, \kappa_{c}^{3}=0.3, \kappa_{c}^{4}=0.2$.

The taxes are defined by $r^{l}\left(p^{l} q^{l}\right):=\omega_{\tau}^{l} p^{l} q^{l}, l=$ $1, \ldots, 4$, where $\omega_{\tau}^{1}=0.03, \omega_{\tau}^{2}=0.2, \omega_{\tau}^{3}=0.01, \omega_{\tau}^{4}=$ 0.1 .

To analyze the usefulness of negotiated transfer pricing in the context of a decentralized multidivisional firm, we first employ the procedure presented in Section 3 for computing the disagreement point. This procedure entails the two divisions initially disagree on a fixed-price $\tilde{p}$ specifying a transfer quantity $\tilde{q}$.

Fixing $p=1, \gamma=5 \times 10^{-8}, \mu=1 \times 10^{-3}$ and $\delta=8 \times 10^{-1}$, and applying

$$
\tilde{f}_{l}\left(\tilde{x}^{l *}, \tilde{x}^{\hat{l}}\right)=\sum_{l=1}^{n}\left|\varphi_{l}\left(\overline{\tilde{x}}^{l *}, \tilde{x}^{\hat{l}}\right)-\varphi_{l}\left(x^{l}, x^{\hat{l}}\right)\right|
$$

for computing the disagreement point, we have that

$$
\begin{gathered}
\tilde{p}=[93500,77000,99000,88000], \\
\tilde{q}=[760,880,1140,770] .
\end{gathered}
$$

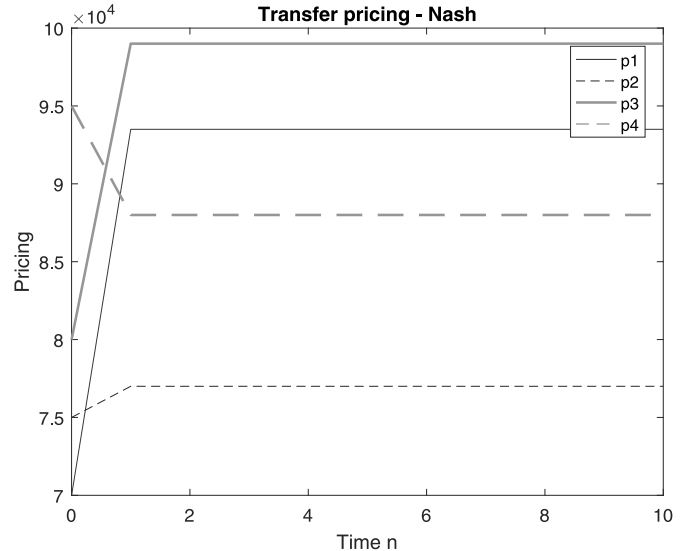

Fig. 5. Disagreement point price $\tilde{p}$.

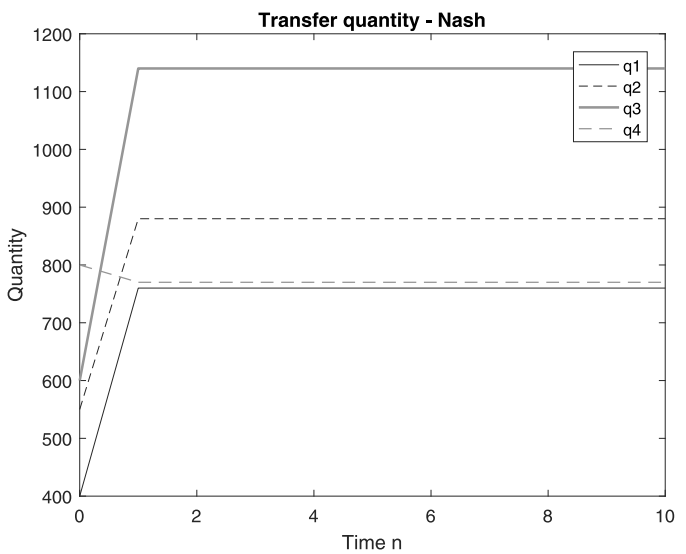

Fig. 6. Disagreement point quantity $\tilde{q}$.

Figures 5 and 6 show the convergence process for the disagreement point.

Then, the initial transfer pricing is renegotiated, and a new trade quantity for $q$ and a new price $p$ are specified.

Now, fixing $\gamma=3.8 \times 10^{-4}, \mu=1 \times 10^{-1}$ and $\delta=2.01 \times 10^{-1}$, and applying Eqn. 26, we obtain

$$
\begin{gathered}
p=[93500,81870,99000,100260], \\
q=[760,880.1,1140,855.3] .
\end{gathered}
$$

Figures 7 and 8 show the new agreement point where all the players improve their positions or remain the same. The resulting process is a simultaneous adjustment of quantity and the transfer price. The renegotiation procedure itself is cooperative in the sense that the divisions jointly maximize the operating profit. The renegotiation utility is defined as the difference between the maximum operating profit and the profit resulting from the initial quantity $\tilde{q}$ as well as the initial transfer price $\tilde{p}$. The conditions ensure that for all the divisions the operating profit under the new equilibrium point improves the payoffs resulting from the renegotiation. Then, it 


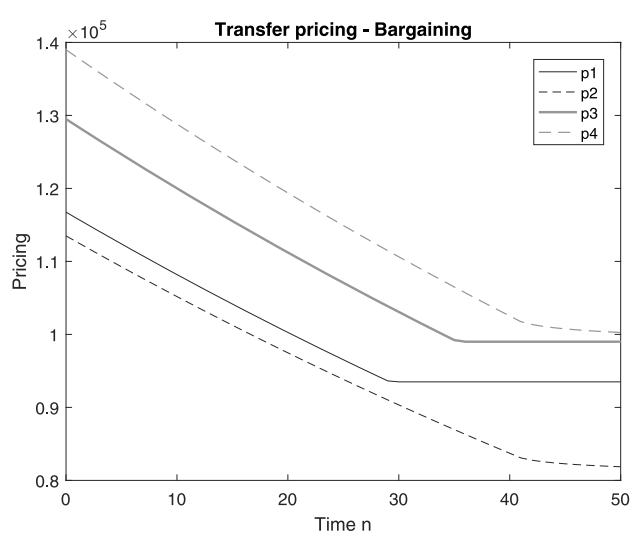

Fig. 7. Agreement point price $p$.

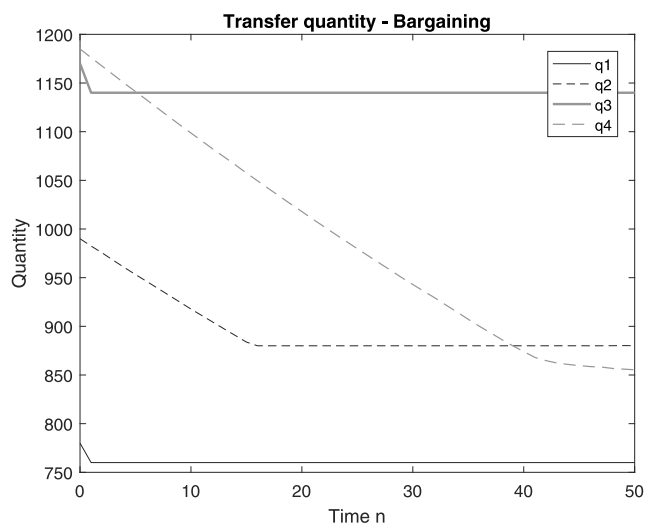

Fig. 8. Agreement point quantity $q$.

may be beneficial for all divisions to adjust price and the transfer quantity in order to find a Pareto improving solution.

\section{Conclusion}

We presented a new solution approach to negotiating the transfer price using the Nash bargaining game theory model for multidivisional firms. We provided a solution model where divisions cooperatively make their decisions to maximize the global profits of the firm. To the best of our knowledge, there are few solutions presented in the literature able to solve the price transfer problem for multidivisional firms employing cooperative game theory, specifically, Nash's bargaining approach for multiple divisions. We proposed a framework and a method based on the Nash equilibrium approach for computing the disagreement point. We introduced the bargaining solution, which is a single-valued function that selects the most preferred alternative within the set of feasible outcomes that produces a profit-maximizing allocation of the transfer price between divisions. We proposed an optimization method for computing the bargaining solution.

In future work, there are many challenging issues remaining to be addressed. Given that in the bargaining game all players improve their positions, we are planning to extend the bargaining concept to a more realistic approach where in negotiations some players need to be better off and others worse off. We will also propose an alternative and improved optimization method for computing the bargaining solution (Clempner and Poznyak, 2011; Shtessel, 1996; Trejo et al., 2015). In addition, we are going to develop an extension of this work applying Stackelberg games.

\section{References}

Abdel-Khalik, A. and Lusk, E. (1974). Transfer pricing a synthesis, Accounting Review 49(1): 8-23.

Alm, J. (2012). Measuring, explaining, and controlling tax evasion: Lessons from theory, experiments, and field studies, International Tax and Public Finance 19(1): 54-77.

Amershi, A. and Cheng, P. (1990). Intrafirm resource allocation: The economics of transfer pricing and cost allocations in accounting, Contemporary Accounting Research 7(1): 61-99.

Arrow, K.J. (1959). Optimization, decentralization, and internal pricing in business firms, Contributions to Scientific Research in Management, Western Data Processing Center, UCLA, Los Angeles, CA, pp. 9-18.

Baldenius, T., Reichelstein, S. and Sahay, S. (1999). Negotiated versus cost-based-transfer pricing, Review of Accounting Studies 4(2): 67-91.

Baumol, W.J. and Fabian, T. (1964). Decomposition, pricing for decentralization and external economies, Management Science 11(1): 1-32.

Beer, S. and Loeprick, J. (2015). Profit shifting: Drivers of transfer (mis)pricing and the potential countermeasures, International Tax and Public Finance 22(3): 426-451.

Besanko, D. and Sibley, D.S. (1991). Compensation and transfer pricing in a principal-agent model, International Economic Review 32(1): 55-68.

Blois, K.J. (1978). Pricing of supplies by large customers, Journal of Business Finance \& Accounting (3): 367-379.

Burton, R.M., Damon, W.W. and Loughrid, D.W. (1974). The economics of decomposition: Re-source allocation vs. transfer pricing, Decision Sciences 5(3): 297-310.

Chalos, P. and Haka, S. (1990). Transfer pricing under bilateral bargaining, The Accounting Review 65(3): 624-641.

Chwolka, A., Martini, J.T. and Simons, D. (2010). The value of negotiating cost-based transfer prices, BuR-Business Research 3(2): 113-131.

Clempner, J.B. (2016). Necessary and sufficient Karush-Kuhn-Tucker conditions for multiobjective Markov chains optimality, Automatica 71: 135-142. 
Clempner, J.B. and Poznyak, A.S. (2011). Convergence method, properties and computational complexity for Lyapunov games, International Journal of Applied Mathematics and Computer Science 21(2): 349-361, DOI: 10.2478/v10006-011-0026-x.

Clempner, J.B. and Poznyak, A.S. (2015). Computing the strong Nash equilibrium for Markov chains games, Applied Mathematics and Computation 265: 911-927.

Clempner, J.B. and Poznyak, A.S. (2016). Convergence analysis for pure and stationary strategies in repeated potential games: Nash, Lyapunov and correlated equilibria, Expert Systems with Applications 46: 474-484.

Clempner, J.B. and Poznyak, A.S. (2017). Multiobjective Markov chains optimization problem with strong Pareto frontier: Principles of decision making, Expert Systems with Applications 68: 123-135.

Dearden, J. (1973). Cast Accounting and Financial Control Systems, Addison Wesley, Reno, NV.

Devereux, M. (2007). The impact of taxation on the location of capital, firms and profit: A survey of empirical evidence, Working paper, No. 702, Oxford University Centre for Business Taxation, Oxford.

Edlin, A.S. and Reichelstein, S. (1995). Specific investment under negotiated transfer pricing: An efficiency result, Accounting Review 70(2): 275-291.

Enzer, H. (1975). The static theory of transfer pricing, Naval Research Logistics Quarterly 22(2): 375-389.

Forgó, F., Szép, J. and Szidarovszky, F. (1999). Introduction to the Theory of Games: Concepts, Methods, Applications, Springer US, New York, NY.

Fredrickson, J.W. (1986). The strategic decision process and organizational structure, Academy ol Management Review 11(2): 280-297.

Ghosh, P., Roy, N., Das, S.K. and Basu, K. (2004). A game theory based pricing strategy for job allocation in mobile grids, Proceedings of the 18th International Parallel and Distributed Processing Symposium, Santa Fe, NM, USA, pp. 82-92.

Grabski, S.V. (1982). Transfer pricing in complex organizations: A review and integration of recent empirical and analytical research, in C. Emmanuel et al. (Eds.), Readings in Accounting for Management Control, Springer, New York, NY, pp. 453-495.

Haake, C.J. and Martini, J.T. (2013). Negotiating transfer prices, Group Decision and Negotiation 22(4): 657-680.

Henderson, B.D. and Dearden, J. (1966). New system for divisional control, Harvard Business Review 44(5): 144-146.

Hirshleifer, J. (1956). On the economics of transfer pricing, Journal of Business 29(3): 172-184.

Hirshleifer, J. (1957). Economics of the divisionalized firm, The Journal of Business 30(2): 96-108.

Jennergren, L.P. (1977). The static theory of transfer pricing, Naval Research Logistics Quarterly 24(2): 373-376.
Johnson, N. (2006). Divisional performance measurement and transfer pricing for intangible assets, Review of Accounting Studies 11(2/3): 339-365.

Kalai, E. and Smorodinsky, M. (1975). Other solutions to Nash's bargaining problem, Econometrica 43(3): 513-518.

Kanodia, C. (1979). Risk sharing and transfer price systems under uncertainty, Journal of Accounting Research 17(1): 74-75.

Karpowicz, M.P. (2012). Nash equilibrium design and price-based coordination in hierarchical systems, International Journal of Applied Mathematics and Computer Science 22(4): 951-969, DOI: 10.2478/v10006-012-0071-0.

Leng, M. and Parlarb, M. (2012). Transfer pricing in a multidivisional firm: A cooperative game analysis, Operations Research Letters 40(5): 364-369.

Markides, C.C. and Williamson, P.J. (1996). Corporate diversification and organizational structure: A resource-based view, Academy of Management Journal 39(2): 340-367.

McAulay, L., Scrace, A. and Tomkins, C. (2001). Transferring priorities: A three-act play on transfer pricing, Critical Perspectives on Accounting 12(1): 87-113.

Nash, J.F. (1950). The bargaining problem, Econometrica 18(2): 155-162.

OECD (2010). OECD Transfer Pricing Guidelines for Multinational Enterprises and Tax Administrations 2010, OECD Publishing, Paris.

Poznyak, A.S. (2008). Advanced Mathematical Tools for Automatic Control Engineers. Deterministic Technique, Vol. 1, Elsevier, Amsterdam/Oxford.

Ronen, J. and Balachandran, K.R. (1988). An approach to transfer pricing under uncertainty, Journal of Accounting Research 26(2): 300-314.

Rosenthal, E.C. (2008). A game theoretic approach to transfer pricing in a vertically integrated supply chain, International Journal of Production Economics 115: 542-552.

Shtessel, Y. (1996). Principle of proportional damages in multiple criteria LQR problem, IEEE Transactions on Automatic Control 41(3): 461-464.

Tanaka, K. (1989). The closest solution to the shadow minimum of a cooperative dynamic game, Computers \& Mathematics with Applications 18(1-3): 181-188.

Tanaka, K. and Yokoyama, K. (1991). On $\epsilon$-equilibrium point in a noncooperative $n$-person game, Journal of Mathematical Analysis and Applications 160(2): 413-423.

Thomas, A. (1980). A behavioral analysis of joint cost allocation and transfer pricing, Technical report, Artbur Andersen \& Co. Lecture Series 1977, Stipes Publishing Company, Champaign, IL.

Trejo, K.K. and Clempner, J.B. (2018). New Perspectives and Applications of Modern Control Theory: In Honor of Alexander S. Poznyak, Springer International Publishing, Cham, (to appear). 
Trejo, K.K., Clempner, J.B. and Poznyak, A.S. (2015). Computing the Stackelberg/Nash equilibria using the extraproximal method: Convergence analysis and implementation details for Markov chains games, International Journal of Applied Mathematics and Computer Science 25(2): 337-351, DOI: 10.1515/amcs-2015-0019.

Trejo, K.K., Clempner, J.B. and Poznyak, A.S. (2017). Nash bargaining equilibria for controllable Markov chains games, 20th World Congress of the International Federation of Automatic Control (IFAC), Toulouse, France, pp. 12772-12777.

Vaysman, I. (1998). A model of negotiated transfer pricing, Journal of Accounting and Economics 25(3): 349-384.

Wahab, O.A., Bentahar, J., Otrok, H. and Mourad, A. (2016). A Stackelberg game for distributed formation of business-driven services communities, Expert Systems with Applications 45(1): 359-372.

Watson, D.J.H. and Baumler, J.V. (1975). Transfer pricing: A behavioral context, The Accounting Review 50(3): 466-574.

Wielenberg, S. (2000). Negotiated transfer pricing, specific investment, and optimal capacity choice, Review of Accounting Studies 5(3): 197-216.

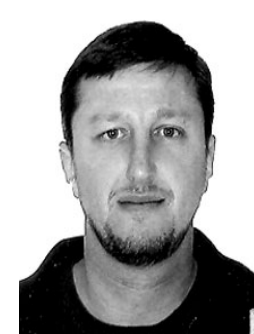

Julio B. Clempner holds a $\mathrm{PhD}$ in computer science from the Center for Computing Research at the National Polytechnic Institute, Mexico. His research interests are focused on justifying and introducing the Lyapunov equilibrium point into game theory. In addition, he is also working on justifying and introducing the manipulation equilibrium point based on Machiavellian social science theory. These interests have lead to several streams of research. One covers the use of Markov decision processes for formalizing the previous ideas. Another stream is related to the use of Petri nets as a language for modeling decision process and game theory. The final direction is optimization and Markov chains. Dr. Clempner is a member of the Mexican National System of Researchers (SNI), and several North American and European professional organizations.

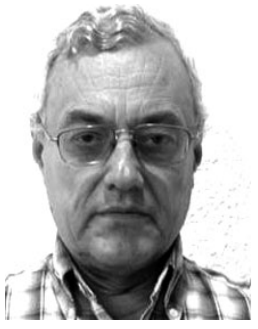

Alexander S. Poznyak graduated from Moscow Physical Technical Institute (MPhTI) in 1970. He earned his $\mathrm{PhD}$ and $\mathrm{DSc}$ degrees from the Institute of Control Sciences of the Russian Academy of Sciences in 1978 and 1989, respectively. From 1973 up to 1993 he served this institute as a researcher and a leading researcher, and in 1993 he accepted the post of a full professor (3-F) at CINVESTAV at the National Polytechnic Institute, Mexico. He is the head of the Automatic Control Department. He has published more than 200 papers in various international journals and 10 books. He is a regular member of the Mexican Academy of Sciences and the System of National Investigators (SNI-3). He is a fellow of the IMA (Institute of Mathematics and Its Applications, UK) and an associated editor of the Journal on Mathematical Control and Information, Kybernetika as well as the International Journal on Computations and Systems.

Received: 30 January 2017

Revised: 19 May 2017

Re-revised: 10 August 2017

Accepted: 1 September 2017 